\title{
Determination of the transfer of tritium to crops fertilised with contaminated sewage sludge
}

\author{
G.J. Ham, D. Wilding and B.T. Wilkins \\ Health Protection Agency, Centre for Radiation, Chemical and Environmental Hazards, \\ OX11 ORQ Chilton, Didcot, Oxon, UK
}

\begin{abstract}
Authorised discharges of radionuclides into sewers can result in contamination of the sludge, and so provide a pathway by which radionuclides can enter the foodchain. Specific data on the uptake of radionuclides from sludge amended land into crops is scarce.

A study has been undertaken to generate data on tritium uptake by crops grown in soil amended with tritium contaminated sewage sludge using a range of soil types and crops common to the UK. The study was performed over two years. The concentration of tritium in soil fell throughout the study. It was therefore not possible to quantify individual soil to crop transfer in terms of conventional concentration ratios. Instead, an aggregated transfer quotient was estimated relating the concentration in the edible part of the crop in $\mathrm{Bq} \mathrm{kg}^{-1}$ to the original amount of activity applied to the soil in $\mathrm{Bq} \mathrm{m} \mathrm{m}^{-2}$. The values obtained were broadly similar at for all crop and soil types studied, being about $210^{-4} \mathrm{~m}^{2} \mathrm{~kg}^{-1}$. The observed values depend on factors such as temperature and rainfall, and so they should not be used in generalised radiological assessments. They are however indicative of the small amounts of tritium transferred to crops from amended soil.
\end{abstract}

\section{INTRODUCTION}

In the UK, the extent to which sewage sludge is used as a soil conditioner is increasing. Authorised discharges of radionuclides into sewers can result in contamination of the sludge, and so together these factors provide a pathway by which radionuclides can enter the food chain. A review of published information, carried out by the National Radiological Protection Board [1], indicated that specific data on the uptake of radionuclides from sludge amended land into crops were scarce. Current discharges from a radiopharmaceutical facility in Cardiff in Wales are presently routed to a new waste water treatment works, and have resulted in elevated concentrations of some radionuclides in sewage sludge pellets. There is therefore the potential to generate specific data on radionuclide uptake by crops grown in soil amended with sludge from this treatment works.

In 2004, the Food Standards Agency (FSA) let a contract to the NRPB to exploit this possible research opportunity. NRPB became the Radiation Protection Division of the Health Protection Agency (RPD) in April 2005. Provided that activity concentrations in the sludge were sufficient, the aim of the work was to make use of the RPD's existing lysimeter facility at Chilton to generate realistic uptake data for selected crops grown in three soil types. This was to be augmented by smaller scale studies on a wider range of crops that would generate relative values for uptake parameters, based on the same soils. Analyses of the sludge pellets showed that only tritium was present in sufficient quantities for the intended study.

Currently, there is very considerable uncertainty about the rate at which tritium can be lost from soil, either via transpiration or via downward migration. This has important implications for situations involving the application of tritium-containing sludge to agricultural land because of the constraints of the Sludge (Use in Agriculture) Regulations (commonly referred to as the Safe Sludge Matrix). These regulations require that crops cannot be harvested from treated arable land until at least 10 months after the sludge has been applied. The aims of the first phase of this project, carried out during 2005, were to determine whether uptake was measurable in crops grown in treated soil and to investigate whether 
tritium was being lost rapidly from the soil. It was recognised that, in terms of crop production, this part of the study was outside the constraints of the Safe Sludge Matrix. The results of this phase of the work were then used to aid the design of the next phase of the work in 2006. The experiments in 2006 focused on uptake into plants under growing conditions that did conform with the Safe Sludge Matrix.

\section{STUDY DESIGN AND INITIATION}

\subsection{Starting materials}

Sewage sludge pellets were collected from the Cardiff East waste water treatment works in November 2004. An initial analysis of the tritium content gave average concentration of about $87,000 \mathrm{~Bq} \mathrm{~kg}^{-1}$ of total tritium. Nearly all of the activity was in what was operationally-defined as an organically bound form. The existing lysimeter facility at the RPD Chilton laboratory is set out in three pairs, with each pair containing a different soil type. The soil types employed are Hamble loam, Fifield sand and Adventurers peat. Each soil was collected from suitable field sites in 1983. Each lysimeter is $1.7 \mathrm{~m}$ in diameter and $0.5 \mathrm{~m}$ in depth.

To enable a larger range of crops and soil types to be studied, the lysimeters were supplemented by a series of smaller containers. Water cisterns of 201 capacity were used with dimensions of $0.45 \mathrm{~m} *$ $0.3 \mathrm{~m} * 0.27 \mathrm{~m}(\mathrm{w} * \mathrm{~d} * \mathrm{~h})$. Soils for these new containers were collected from the original locations in 2005. In addition, samples of a clay soil of the Denchworth series were also collected. Together, these four soils provide a suitable contrast in the types of agricultural soil found across the UK.

Drainage holes were drilled in each of the new containers and after each container had been filled, the soil was tilled and then allowed to settle. At about the same time the soils in the established lysimeters were dug over and allowed to settle.

\subsection{Approach adopted}

The crops chosen for the study were dwarf french (DF) bean, cabbage, carrot, strawberries, potatoes and grass. Each soil : crop combination involved 3 of the smaller containers. Two of these were treated with sludge pellets; the third remained untreated and was used as a control. This was considered important because of the possibility of transpiration, ie the transfer of tritium in the vapour phase between containers. This approach also provided a check that ambient levels of tritium in the environment were not affecting the results. All crops except the strawberries were also grown in the lysimiters. All crops except the strawberries and grass were rotated between the containers and lysimiters for the second year of the study so they were not grown in the same tub two years running.

Once the soil had settled after tillage, the designated containers and the six lysimeters were amended with sewage sludge pellets at a rate corresponding to about 20 tonnes per hectare $\left(2 \mathrm{~kg} \mathrm{~m}^{-2}\right)$ which is equivalent to $1.710^{5} \mathrm{~Bq} \mathrm{~m}^{-2}$ of tritium. This exceeded the maximum rate specified in the Safe Sludge Matrix, but was considered necessary because the measured concentrations of tritium in the pellets were lower than had originally been expected. The pellets were incorporated into the top $100 \mathrm{~mm}$ of soil using standard gardening tools. The soils were mixed again 3 and 7 days after amendment.

For 2005, one important objective was to improve understanding of the behaviour of tritium in soil. For this reason, samples of soil were taken from the lysimeters throughout the growing season. The results will be discussed in detail later but the rate of change in the concentration of tritium in soil decreased through 2005. For this reason, in 2006 soil was only sampled at the beginning and end of the growing season. One sample of each soil type was also taken from the lysimeters in June 2007, to investigate the effects of a second winter. Soils in the smaller containers were sampled only before planting or sowing and then again when a crop was harvested. After collection, all soil samples were sieved through a mesh of approximately $3 \mathrm{~mm}$ in size. One aliquot was dried at $105^{\circ} \mathrm{C}$ to determine moisture content and a second aliquot stored at $-20^{\circ} \mathrm{C}$ for tritium analysis. The smaller containers provided an 
opportunity to collect and analyse leachate, ie the liquid that had percolated through the soil and was drained away. One container of each soil type that had been sown with grass was used for this purpose.

Crops were sown or planted in late May 2005 for the first phase and in early May 2006 for the second phase. With the exception of the permanent crops (grass and strawberries), crops were rotated between containers over the two years. They were maintained according to good horticultural practice, watered as required and regularly fed with a proprietary liquid feed. Grass was cut to about $30 \mathrm{~mm}$ above the soil surface whenever it reached about $150 \mathrm{~mm}$ in height. All other crops were collected at maturity. All crops except grass were washed in tap water and inedible parts discarded. The entire sample of the edible part was then roughly chopped or shredded. An aliquot was stored at $-20^{\circ} \mathrm{C}$ for tritium analysis. Potatoes and carrots were not peeled and were analysed as complete tubers or roots. Analyses were carried out using a commercial combustion apparatus and a low level liquid scintillation counter. In 2005, an additional sample of one potato crop was taken from a lysimeter. This was used to determine the distribution of activity between peel and flesh. For several other radionuclides, the concentration in the peel is greater than in the flesh [2], and such information is therefore valuable when specific radiological assessments are required. In 2005, the lysimeters were able to produce more than enough mature crop for the subsequent analyses, and so in 2006 a wider range of crops was grown in them, under conditions that were more representative of those in the field.

\section{RESULTS AND DISCUSSION}

With the exception of strawberries in 2005 , all crops produced sufficient material for analysis. The growing season in 2006 was exceptionally hot and dry and therefore all crops required extensive watering with tap water. Despite this, very little leachate was collected from the containers set up for this purpose. In addition, yields of all crops were lower than in 2005.

\subsection{Concentrations and movement of tritium in soil}

The results for total and aqueous tritium concentrations in surface soil from the lysimeters and containers showed a large variability in the individual results. However, this variability tended to decrease with time after the pellets were added. In all cases the tritium was predominately present as the organically bound form. As an example the data for the lysimeter loam soils are shown in Figure 1.

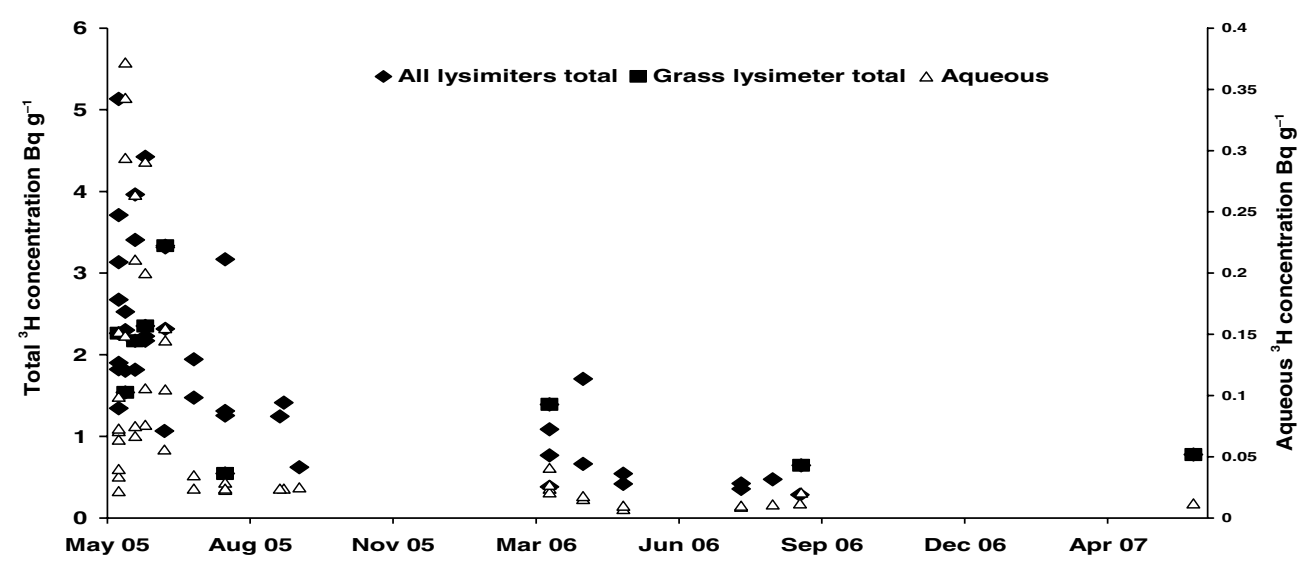

Figure 1. Concentrations of tritium in loam soil.

The reproducibility of the analytical method had already been established, and so the variability can probably be ascribed to the non-uniform distribution of sludge in the soil. 
The soils used for growing grass were the only ones that were not disturbed by cropping or tilling between the two years. For this reason, these results are differentiated in Figure 1. However the data for these soils did not appear to be less variable than the other results.

The non-uniform distribution of the sludge pellets in the soil in the early stages of the study mean that only very broad comments can be made about temporal trends. The inhomogeneity decreased with time but the ranges of individual values from the same soil were still large. Table 1 shows the mean decrease in tritium concentrations over the two growing seasons. Given the spread of the individual data for the sand soil in May and August/September 2006, the mean values for 2005 and 2006 were not significantly different from each other.

Table 1. Mean loss of tritium from soils.

\begin{tabular}{|c|c|c|c|c|c|c|c|}
\hline \multicolumn{8}{|c|}{ Percent loss of tritium in each growing season } \\
\hline & \multicolumn{3}{|c|}{ Lysimiters } & \multicolumn{4}{|c|}{ Containers } \\
\hline & Loam & Peat & Sand & Loam & Peat & Sand & Clay \\
\hline 2005 & $58 \%$ & $34 \%$ & $55 \%$ & $65 \%$ & $27 \%$ & $67 \%$ & $67 \%$ \\
\hline 2006 & $44 \%$ & $33 \%$ & $-17 \%^{1}$ & $32 \%$ & $34 \%$ & $11 \%$ & $42 \%$ \\
\hline Whole study & $82 \%$ & $81 \%$ & $71 \%$ & $84 \%$ & $71 \%$ & $83 \%$ & $87 \%$ \\
\hline
\end{tabular}

In August 2005 and April $2006300 \mathrm{~mm}$ deep cores were taken from the undisturbed part of the lysimiters. The loam and clay soils showed no evidence of migration of the activity from the top $100 \mathrm{~mm}$ layer, while there was some evidence that tritium was present in the 100-200 and 200-300 mm layers of the peat soil.

As previously mentioned one container of each soil type was modified to collect leachate. In 2005 the activity in the leachate accounted for about $10 \%$ of the applied tritium for the peat soil and about $25 \%$ for loam, sand and clay. As mentioned earlier, in 2006 very little leachate was collected and in all cases it only represented about $1 \%$ of the applied tritium. In all cases the tritium was predominately present in the aqueous form. Despite the uncertainties and variability in the measurements, the losses of tritium from the soil cannot be fully explained by the tritium in the leachate.

\subsection{Transfer of tritium into vegetables}

The measured concentrations for crops grown in untreated soils were generally very low, many being below the limit of detection. These results implied that any cross-contamination via transpiration from treated soils and crops was small. They also suggested that any effects from the presence of ambient levels of tritium in the general environment were small.

All measured values for vegetables grown in treated soils were low, with large associated counting uncertainties. Generally, however, where analyses were carried out on the same crop and soil type in two separate containers, the results were not significantly different from each other. In most cases the tritium in vegetables was nearly all present as tritiated water.

The absence of OBT in many samples of vegetables deserves further comment, because tritium should become incorporated into organic matter as a result of photosynthesis. The moisture content of the crops was in the range 80-95\%. On the basis of the specific activity approach the ${ }^{1} \mathrm{H}:{ }^{3} \mathrm{H}$ ratio in the plant water and organic matter should be the same [3]. Taking the relatively low percentage of organic matter in fresh vegetables with the very low concentrations of tritiated water in some of these samples, then concentrations of tritium in the form of OBT might be below the detection limit. The sample of potato that was peeled showed no significant difference in tritium content between the peel and flesh. Table 2 summarises the average concentration of tritiated water in crops.

For many radionuclides, uptake from soil into plants has been defined using the soil - plant transfer factor approach, i.e. the quotient of the activity concentration in the plant to that in the soil. However, 
Table 2. Average concentration of tritiated water in crops.

\begin{tabular}{|l|l|l|l|l|l|l|l|}
\hline \multicolumn{2}{|c|}{ Soil } & Experiment & \multicolumn{2}{l|}{ Tritium concentration, Bq g } \\
\hline \multicolumn{2}{|l|}{} & & Cabbage & Carrots & DF Beans & Potatoes & Strawberries \\
\hline Clay & 2005 & Container & 0.035 & 0.025 & 0.04 & 0.04 & \\
\hline & 2006 & Container & $<0.007$ & 0.03 & 0.023 & 0.010 & 0.014 \\
\hline Loam & 2005 & Container & 0.05 & 0.016 & 0.05 & 0.035 & \\
\hline & & Lysimeter & & 0.10 & & 0.12 & \\
\hline & 2006 & Container & 0.01 & 0.03 & 0.029 & 0.016 & 0.015 \\
\hline & & Lysimeter & 0.033 & 0.04 & 0.05 & 0.024 & \\
\hline Peat & 2005 & Container & 0.025 & 0.03 & 0.05 & 0.05 & \\
\hline & & Lysimeter & & 0.12 & & 0.08 & \\
\hline & 2006 & Container & $<0.008$ & 0.02 & 0.023 & 0.027 & 0.017 \\
\hline & & Lysimeter & 0.032 & 0.03 & 0.039 & 0.057 & \\
\hline Sandy & 2005 & Container & 0.05 & 0.05 & 0.05 & 0.07 & \\
\hline & & Lysimeter & & 0.11 & & 0.20 & \\
\hline & 2006 & Container & 0.01 & 0.03 & 0.033 & 0.033 & 0.015 \\
\hline & & Lysimeter & 0.048 & 0.02 & 0.037 & 0.040 & \\
\hline
\end{tabular}

concentrations of tritiated water and OBT in surface soil changed rapidly during the 2005 growing season, making the transfer factor approach unsuitable for this study. An aggregated transfer quotient approach is more helpful as it provides an indication of the overall transfer of tritium to crops from the activity originally applied. For this purpose, a quotient has been estimated that relates the concentration in the edible part of the crop in $\mathrm{Bq} \mathrm{kg}^{-1}$ fresh mass to the original amount of activity applied to the soil in $\mathrm{Bq} \mathrm{m}^{-2}$. This quotient would be affected by factors such as temperature and rainfall, and so would be specific to this study. Consequently, the numerical values of such quotients should not be applied more generally in radiological assessments. Here, the aggregated transfer quotients were broadly similar between crop and soil types. All were typically about $210^{-4} \mathrm{~m}^{2} \mathrm{~kg}^{-1}$, which illustrates how little of the activity originally applied was transferred to crops.

\subsection{Grass}

In contrast to the vegetable crops, where most of the activity was in the form of tritiated water, the results for grass indicated a significant and variable contribution from OBT. As in the case of arable crops, grass has a high moisture content and so the amount of OBT deriving solely via root uptake from the soil was likely to be close to or below detection limits. The amounts of OBT observed in grass therefore suggested that a considerable proportion of the measured tritium activity was due to soil being splashed on to the vegetation. Taking broad averages, the OBT content of the grass could be accounted for by the presence of a few percent by mass of the underlying soil.

The results for grass, together with the retention of OBT in the surface soil, have implications for predictions of the onward transfer of activity into grazing animals. For many radionuclides, the inadvertent ingestion of soil during grazing can be an important route of intake of activity [4]. The results from this study indicate that for tritium, soil ingestion could be important when new pasture is sown on land that has been treated with sludge. This is because uptake of tritium as tritiated water from the soil is relatively low while a reservoir of activity remains in the surface soil in the general form of OBT. The availability of tritium in an organically-bound form for onward transfer to milk or meat would be difficult to quantify since it could depend on the actual chemical species involved.

\section{CONCLUSIONS}

Concentrations of tritium in treated soil decreased throughout the period of the experiment. Measured values in the soil itself displayed considerable variability, although this decreased with time. 
In broad terms about $80 \%$ of the applied tritium was lost over the two growing seasons. The tritium that remained in the soil was mostly in the organically bound form and retained in the surface $(0-100 \mathrm{~mm})$ layer. While tritium was measured in the leachate from containers it could only account for under half of the tritium lost from the soil. Therefore it seems likely that tritium was lost by transpiration either directly from the soil or through the plants.

Concentrations of tritium were measurable in nearly all crops grown in treated soils, but the actual values were generally low. With the exception of grass, for which soil splash seemed to be important, the activity was mainly in the form of tritiated water. The changes in concentrations of tritium in soil over the growing seasons and the low concentrations measured in crops meant that it was not possible to quantify individual soil to crop transfer in terms of the conventional soil - plant transfer approach. However, an aggregated transfer quotient approach has been used to relate the concentration in the fresh edible parts of crops grown in 2006 to the amount of activity originally applied to the soil in 2005 . The aggregated transfer quotients were typically around $210^{-4} \mathrm{~m}^{2} \mathrm{~kg}^{-1}$, indicating that transfer of tritium from sewage sludge to crops is likely to be small.

\section{Acknowledgments}

This study was financed by the UK Food Standards Agency under contract number R0106. A detailed report of the study can be obtained on the HPA intranet site [5].

\section{References}

[1] Ham G.J., Shaw S., Crockett G.M. and Wilkins B.T., Partitioning of radionuclides with sewage sludge and transfer along terrestrial foodchain pathways from sludge-amended land - a review of data. Chilton, (National Radiological Protection Board, Chilton UK, 2003) NRPB-W32.

[2] Green N., Wilkins B.T. and Poultney S., J Radioanalytical and Nuclear Chemistry, 226 (1997) $75-78$.

[3] IAEA., Generic models for use in assessing the impact of discharges of radioactive substances to the environment. IAEA, Vienna, 2001, Safety Report Series No 19.

[4] Green N., Johnson D. and Wilkins B.T., J Environ Radioact, 30 (1996), 173-183.

[5] Ham G.J., Wilding D. and Wilkins B.T., Tritium concentrations in crops fertilised with contaminated sewage sludge. (Radiological Protection Division, Health Protection Agency. Chilton UK, 2007) HPA-RPD-038. 